\title{
Predictive Value of Cerebrospinal Fluid Biomarkers for Tap Test Responsiveness in Patients With Suspected Idiopathic Normal Pressure Hydrocephalus
}

\section{Rongrong Hua}

Department of Neurology,Aviation General Hospital

Chunyan Liu

Department of Neurology, Aviation General Hospital

Xing Liu

Department of Neurology, Aviation General Hospital Jinwu Zhu

Aviation Medical Engineering Center of Aviation General Hospital

Jie Zhang

Department of Neurology, Aviation General Hospital

\section{Lidong Wang}

Department of Neurology, Aviation General Hospital

\section{Zhe Shi}

Department of Neurology, Aviation General Hospital

\section{Jian Li}

Department of Neurology, Aviation General Hospital

\section{Shuangyan Kong}

Department of Neurology, Aviation General Hospital

\section{Chenhui Yang}

Department of Neurology, Aviation General Hospital

Nan Liu

Department of Neurology, Aviation General Hospital

Lijuan Liu

Department of Neurology, Aviation General Hospital Jie Sun

Department of Neurology, Aviation General Hospital

\section{Qiong Yang}

Department of Neurology, Aviation General Hospital

\section{Yubing Wu}

Department of Neurology, Aviation General Hospital 


\section{Ying Zhou}

Department of Neurology, Aviation General Hospital

\section{Yanfeng Li}

Department of Neurology, Peking Union Medical College Hospital

Yan Xing ( $\sim$ hkzyysjnk@sina.com )

activation general hospital https://orcid.org/0000-0002-4860-5709

\section{Research}

Keywords: idiopathic normal pressure hydrocephalus, cerebrospinal fluid biomarkers, tap test

Posted Date: January 13th, 2021

DOI: https://doi.org/10.21203/rs.3.rs-143397/v1

License: (c) (1) This work is licensed under a Creative Commons Attribution 4.0 International License.

Read Full License 


\section{Abstract}

Background The value of cerebrospinal fluid (CSF) biomarkers for idiopathic normal pressure hydrocephalus (iNPH) needs to be determined. This prospective study aimed to reveal the correlation between CSF biomarkers and clinical symptoms of iNPH, and its predictive value for tap test responsiveness.

Case presentation Thirty-nine suspected iNPH patients were recruited, contributed qualified CSF, and accepted a tap test and unified pre- and post-test evaluation of neurological function. The analysis of biomarkers from their CSF showed a decrease of tau and its phosphorylated form, especially in the tap test (+) group. In addition, the responsiveness of the tap test was also related to the number of combined symptoms $(p<0.01)$. A correlation was also found between the end pressure or pressure difference of CSF and tap test responsiveness $(p<0.05)$. The results of binary logistic regression analysis showed that $P$ (tap test responsiveness $)=1 / 1+\mathrm{e}^{\wedge}-(-5.505+55.314$ * ratio of $\mathrm{p} / \mathrm{T}$-tau -1.586 * numbers of combined symptoms). The combined indicators $(-5.505+0.553 *$ percentage of $\mathrm{p} / \mathrm{T}$-tau -1.586 * numbers of combined symptoms) gave the highest sensitivity and specificity, which were $94.12 \%$ and $72.73 \%$, respectively.

Conclusions The combined indicators calculated from $\mathrm{p} / \mathrm{T}$-tau and the numbers of combined symptoms may be accessed in judgment of tap test responsiveness, which is beneficial for the feasibility of clinical application.

\section{Introduction}

Idiopathic normal pressure hydrocephalus (iNPH) is a critical brain disorder without a clear cause that occurs in adults and is accompanied by excess cerebrospinal fluid (CSF) accumulation in the ventricular system, resulting in gait dysfunction, a frontal-subcortical pattern of cognitive impairment, or urinary urge incontinence with insidious onset $[1,2]$. The pathophysiology of iNPH is most likely multifactorial through cerebrospinal fluid dynamic disturbance [3], although clinicians are more likely to combine the key clinical symptoms and imaging findings to diagnose iNPH. Shunt placement is recommended as an effective treatment for patients with iNPH, although large double-blind studies are still necessary [4]. It is possible that $60 \%-80 \%$ of patients could benefit from shunt surgery [5]. Unfortunately, iNPH has no reliable biomarker to assist in the selection of patients who could benefit from shunt surgery.

Researchers recently reported data indicating that the presence of amyloid beta 1-42 (Aß1-42), the pathologic hallmark of $A D$ in cortical biopsies obtained at the time of shunt placement, is associated with poorer response to shunting in patients with iNPH [6]. Although quantifications of CSF biomarkers, such as $A \beta 1-42$, total tau ( $\mathrm{T}$-tau), and phosphorylated tau ( $\mathrm{p}$-tau) proteins, have been incorporated into standard diagnostic guidelines for degenerative diseases such as $A D$, there is also a need to explore potential biomarkers for the diagnosis of hydrocephalus and responsiveness of receiving a shunt. In addition, the neurodegenerative markers T-tau, ${ }^{181} \mathrm{p}$-tau and A $\beta 1-42$ successfully differentiate between 
$\mathrm{AD}$ and $\mathrm{iNPH}$ and therefore may be candidate biomarkers for prognosis and shunt response in iNPH [7]. However, the details are still unclear. The aim of this study was to explore the related factors of the tap test and the predictive value of CSF T-tau, ${ }^{181} \mathrm{p}$-tau and $\mathrm{A} \beta 1-42$ for shunt responsiveness.

\section{Methods}

\section{Patients}

The study protocol was approved by the Ethics Committee of Aviation General Hospital. All methods were performed in accordance with the relevant guidelines and regulations $[8,9]$. Patients were recruited consecutively from the neurology department at Aviation General Hospital, China Medical University from January 1, 2017 to August 1, 2019. The inclusion criteria were age $>60$ years and iNPH diagnosed according to the guidelines. The available medical information was obtained from medical records and treating physicians. All patients $(n=42)$ underwent standard screening at baseline, including physical and neurological examination, magnetic resonance imaging (MRI), and laboratory tests. Diagnoses were made by consensus in a multidisciplinary team without knowledge of CSF results. Informed consent was obtained from all participants and/or their legal guardians. All subjects gave written informed consent for the use of clinical data for research purposes.

\section{Tap test and CSF collection}

Lumbar puncture should preferably be performed in the morning. After a routine examination excluded surgical contraindications, an intervertebral lumbar puncture was performed at L3-4 or L4-5 under local infiltration anesthesia. After confirming that the needle had entered the subarachnoid space, the initial pressure of CSF was determined. Then, the cerebrospinal fluid was collected and placed in two $15 \mathrm{~mL}$ polypropylene centrifuge tubes (430791, Corning, New York, USA), which were immediately centrifuged \pm $3700 \mathrm{~g}$ for 10 minutes at $4{ }^{\circ} \mathrm{C}$ to exclude insoluble materials. Then, the fluid was dispensed into $1 \mathrm{ml}$ polypropylene tubes and stored at $-80^{\circ} \mathrm{C}$. After measuring the end pressure of CSF and removing the lumbar puncture needle, the tap test was complete. Laboratory staff was blinded to the clinical information of each subject.

\section{CSF analysis}

CSF was sampled for T-tau, p-tau and A $\beta 1-42$ during the reservoir tap test. CSF samples were collected under sterile conditions into $15 \mathrm{~mL} * 2$ centrifuge tubes. CSF sample collection and storage methods were all in accordance with the consensus guidelines for CSF biobanking [21]. Samples were sent for enzymelinked immunosorbent assay (ELISA) analysis to measure concentrations of T-tau (INNOTEST hTAU Ag ELISA kit, Fujirebio Europe N.V., Belgium), ${ }^{181}$ p-tau (INNOTEST PHOSPHO-Tau (181p) ELISA kit, Fujirebio Europe N.V., Belgium), and A $\beta$-42 [INNOTEST $\beta$-AMYLOID (1-42) ELISA kit, Fujirebio Europe N.V., Belgium] and total protein. A technician, blinded to the clinical results, prospectively recorded levels of T-tau, ${ }^{181} \mathrm{p}$ tau, and $A \beta 1-42$. Longitudinal stability in the measurements was ascertained using an elaborate 
program of internal quality control (QC) samples. Intra- and inter-assay coefficients of variation were 1.37 and $5.8 \%$ for $A \beta 1-42,2.51$ and $1.46 \%$ for T-tau, 3.36 and $1.52 \%$ for $p$-tau, respectively.

\section{Standard reference: clinical outcome}

The clinical outcome groups 'tap test positive' or 'tap test negative' provided the reference standard. Outcome measures were recorded prospectively and analyzed retrospectively. Three main outcome objective measures were analyzed: (1) Mini-mental state examination (MMSE) neuropsychology report (a minimum of $10 \%$ improvement observed in time orientation, location orientation, immediate memory, attention and computational power, delayed memory, language, visual space), (2) timed 10-m walking test (a minimum of $5 \%$ improvement in either time in seconds or number of steps, or both) and (3) bladder control (with improvement being the reduction of episodes of incontinence per day of 1 or less). Assessments were performed by personnel blinded to the index test result. For outcome analysis, a 'tap test positive $(+)$ ' reflected better outcome in at least one of the three objective measures after the tap test, in addition to reported subjective improvement. A deterioration in any one of these clinical elements resulted in an overall outcome of 'tap test negative (-).' All outcome data were processed on an anonymous database.

\section{Statistical analysis}

Data were expressed as mean \pm standard deviation or an absolute number with a proportion for descriptive statistics. Continuous variables from two groups were compared using an independent $t$-test. Continuous variables from more than two groups were compared using one-way ANOVA. A rank sum test was used for comparison of non-normal distribution data of pressure difference of CSF between tap (+) and (-) groups. The conditional count data was compared using a chi-square test. Spearman or Pearson correlation analysis was applied to examine the correlations. A binary logistic regression analysis was used to select and clarify the contribution of the related factors to the tap test responsiveness. ROC curves were analyzed, and a cut-off value was selected. A $p$ value of 0.05 was defined as the threshold of statistical significance in each test.

\section{Results}

\section{Study profile}

From January 1, 2017 to August 1, 2019, a total of 39 CSF samples were analyzed from 42 suspected iNPH patients: 6 women and 33 men of mean age $71.44 \pm 8.10$ (range 52-85) years. Figure 1 outlines the study profile.

\section{The levels of lumbar AB1-42, T-tau, and p-tau, and their relationship with tap test response or numbers of combined symptoms}

Previous studies have shown that iNPH patients tended to exhibit low levels of CSF T-tau, and this can be a good predictor of post-operative outcome. Similarly, in our study, the levels of tau and its 
phosphorylated form were significantly decreased, especially in the tap test $(+)$ group $(p<0.01$ and $p<$ $0.05)$, although the levels of $A \beta 1-42$ did not change significantly (Fig. $2 A$ ). The levels of $A \beta 1-42$ in the tap test $(+)$ and $(-)$ groups were $584.86 \pm 303.55 \mathrm{pg} / \mathrm{mL}(\mathrm{n}=17)$ and $641.36 \pm 255.38 \mathrm{pg} / \mathrm{mL}$, respectively $(n=22)($ Fig. 2A). The level of T-tau in the tap test $(+)$ and $(-)$ groups was $191.21 \pm 80.52 \mathrm{pg} / \mathrm{mL}(n=17)$ and $382.88 \pm 25.35 \mathrm{pg} / \mathrm{mL}$, respectively $(n=22)$ (Fig. $2 A)$. The level of $p$-tau in the tap test $(+)$ and $(-)$ groups was $32.38 \pm 12.63 \mathrm{pg} / \mathrm{mL}(n=17)$ and $43.38 \pm 14.02 \mathrm{pg} / \mathrm{mL}$, respectively $(n=22)(F i g .2 A)$. In contrast, the ratio of $\mathrm{p} / \mathrm{T}$-tau in the tap test $(+)$ group was $17.23 \pm 0.59 \%$, which was higher than that in tap test $(-)$ group $(12.81 \pm 0.90 \%)(p<0.001)$ (Fig. 2B). The results suggested that the magnitude of $p$-tau reduction is significantly lower than T-tau, resulting in a relatively higher ratio of $p / T$-tau. In addition, the ratio of $p$-tau/A $A 1-42$ was $6.87 \pm 0.95 \%$ and $7.97 \pm 1.09 \%$ in the tap test $(+)$ and $(-)$ groups $(12.81 \pm$ $0.90 \%)$, respectively $(p>0.05)$ (Fig. 2 C).

To analyze the relationship between CSF biomarkers and the number of combined symptoms of iNPH patients, a correlation analysis was performed. Although the $p$ values of correlation analysis between the actual values of $A \beta 1-42$, T-tau, $p$-tau or $p / T$-tau with the number of combined symptoms were 0.777 , $0.076,0.051$ and 0.568 , respectively, a significant difference in the number of combined symptoms with p-tau/A $\beta 1-42$ was found $(p=0.030)$ (Fig. 2D-H).

A subgroup analysis was used to determine the accurate significant difference of each CSF biomarker in groups with one/two/three combined symptoms. Unfortunately, no difference was found between the subgroups with different numbers of combined symptoms and $A \beta 1-42$, T-tau and $\mathrm{p} / \mathrm{T}$-tau $(p>0.05, p>$ 0.05 and $p>0.05$ ) (Fig. 3A, B and D). The levels of $A \beta 1-42$ in the subgroup with one/two/three combined symptoms were $554.31 \pm 300.82,692.15 \pm 247.35$ and $550.73 \pm 290.23 \mathrm{pg} / \mathrm{mL}$, respectively (Fig. 3A). The levels of T-tau in the subgroup with one/two/three combined symptoms were $192.01 \pm 130.78,311.21 \pm$ 162.83 and $348.92 \pm 231.75 \mathrm{pg} / \mathrm{mL}$, respectively (Fig. 3B). The ratio of $\mathrm{p} / \mathrm{T}$-tau in the subgroup with one/two/three combined symptoms was $0.16 \pm 0.03,0.14 \pm 0.04$ and $0.15 \pm 0.05$, respectively (Fig. 3D). However, the levels of $p$-tau in the subgroup with one/two/three combined symptoms were $29.50 \pm 17.89$, $39.70 \pm 13.34$ and $42.65 \pm 11.81 \mathrm{pg} / \mathrm{mL}$, respectively, which was significantly different between the group with one and three combined symptoms $(p<0.05)$ (Fig. 3C). The ratio of $p$-tau/A $\beta 1-42$ in the subgroup with one/two/three combined symptoms was $0.06 \pm 0.03,0.06 \pm 0.03$ and $0.10 \pm 0.06$, respectively, which was significantly different between the group with one/two and three combined symptoms $(p<0.05$ and $p<0.05$ ) (Fig. 3E). In addition, the responsiveness of the tap test was also related to the number of combined symptoms (Fig. 3F). The numbers of one/two/three combined symptoms in the tap test (+) and (-) were $7 / 6 / 4$ and $1 / 12 / 9$, respectively. Significantly more combined symptoms were obtained by the patients in the tap test $(-)$ group $(p<0.01)$.

\section{The distribution of combined symptoms and its correlated factors}

We showed that patients with a fewer number of combined symptoms may have a positive response to the tap test. Further analysis revealed that the prevalence of hypertension, initial pressure and pressure difference of CSF were also related to the number of combined symptoms. There were significant 
differences in the distribution of combined symptoms of patients with hypertension or not $(p<0.01)$. The number of hypertensive patients with one/two/three combined symptoms was $0 / 14 / 8$, which was $8 / 4 / 5$ in non-hypertensive patients (Fig. 4A). This finding suggested that patients with hypertension would have more symptoms of iNPH than those without hypertension. The initial pressure of CSF in patients with two symptoms of iNPH was $167.22 \pm 45.45 \mathrm{mmH}_{2} \mathrm{O}$, significantly higher than those with three symptoms $\left(125.92 \pm 18.67 \mathrm{mmH}_{2} \mathrm{O}\right)$, which was similar to those with one symptom $\left(151.50 \pm 25.51 \mathrm{mmH}_{2} \mathrm{O}\right)$ (Fig. 4B). The pressure difference of patients with two symptoms of iNPH was $82.22 \pm 48.63 \mathrm{mmH}_{2} \mathrm{O}$, significantly higher than those with one symptom $\left(49.88 \pm 10.63 \mathrm{mmH}_{2} \mathrm{O}\right)$ or three symptoms $(49.77 \pm$ $\left.18.75 \mathrm{mmH}_{2} \mathrm{O}\right)(p<0.05$ and $p<0.05)$, although the end pressure of CSF had no significant difference in iNPH patients with different numbers of combined symptoms (Fig. $4 \mathrm{C}$ and $\mathrm{D}$ ).

\section{The responsiveness of the tap test and its correlated factors}

In addition to combined symptoms, a correlation was also found between the end pressure or pressure difference of CSF and tap test responsiveness $(p<0.05)$, although there was no significant correlation between tap test responsiveness and initial pressure of CSF (Fig. 5A and B). The initial pressure of CSF was $145.12 \pm 22.49 \mathrm{mmH}_{2} \mathrm{O}(\mathrm{n}=17)$ and $154.18 \pm 47.73 \mathrm{mmH}_{2} \mathrm{O}(\mathrm{n}=22)$, which was similar. The end pressure was $96.35 \pm 27.74 \mathrm{mmH}_{2} \mathrm{O}(\mathrm{n}=17)$ in the tap test $(+)$ group, which was significantly higher than that in the tap test (-) group $\left(77.05 \pm 25.24 \mathrm{mmH}_{2} \mathrm{O}, \mathrm{n}=22\right)(p<0.05)$ (Fig. 5A). The median value of pressure difference of CSF was $50 \mathrm{mmH}_{2} \mathrm{O}$ in the tap test (+) group, which was lower than $60 \mathrm{mmH}_{2} \mathrm{O}$ in the tap test $(-)$ group $(p<0.01)$ (Fig. $5 \mathrm{~B})$. The finding suggests better brain tissue compliance in patients who were tap test positive.

To clarify the contribution of these related factors to tap test responsiveness, we conducted further binary logistic regression analysis. The results showed that $P$ (tap test responsiveness) $=1 / 1+e^{\wedge}-(-5.505+$ 55.314 * ratio of $\mathrm{p} / \mathrm{T}$-tau -1.586 * numbers of combined symptoms), suggesting a predictive value of a higher ratio of $\mathrm{p} / \mathrm{T}$-tau and fewer combined symptoms. Inferential combined indicators were calculated equal to $-5.505+0.553 *$ percentage of $\mathrm{p} / \mathrm{T}$-tau -1.586 * numbers of combined symptoms.

Results of the actual value or ratio of p-tau/T-tau and the combined indicators were further analyzed using ROC curves to determine the sensitivity, specificity, and area under the ROC curve (AUC). The combined indicators gave the highest AUC of 0.90 , compared with T-tau (0.87), p-tau (0.63), and p/T-tau (0.82) (Fig. $5 \mathrm{C}$ and D). The cut-off value (-0.77) was selected involving maximizing Youden's Index (0.67), of which the sensitivity and specificity were $94.12 \%$ and $72.73 \%$, respectively.

\section{Discussion}

The latest criteria concerning $A D$ added some reliable biomarkers based on clinical symptoms, such as core amyloid biomarkers and $A \beta$ peptide levels in CSF, which supported its importance and predictability in the diagnosis of neurodegenerative diseases [10]. Moreover, more hyperphosphorylated tau has also been found in the brain and CSF of AD patients, compared with that in normal individuals, and this 
protein participates in microtubule assembly and stability [11]. There is evidence that $A \beta$ and tau in particular are involved in the pathophysiology of other neurodegenerative diseases, and there are differences in levels in different diseases [12-14]. Patients with iNPH consistently appear to have lower CSF T-tau or A $\beta 1-42$ and higher p-tau $[7,15,16]$. The measures of CSF biomarkers of AD were considered not only potential biomarkers to readily distinguish patients with $A D$ from those with iNPH but also predictive factors for shunt responsiveness. Our previous meta-analysis also showed the comparative change of CSF $A \beta 1-42$, T-tau and p-tau in patients with iNPH or AD, compared with the healthy normal state [17]. However, some patients with iNPH with co-existing neurodegenerative disorders had increased CSF T-tau [16], although these patients could also benefit from a ventriculoperitoneal shunt, which reduces the specific diagnostic value of tau and increases the difficulty of diagnosis and prognosis [18].

Unexpectedly, further studies showed that T-tau is greatly affected by changes in cerebrospinal fluid hydrodynamics, while p-tau, especially ${ }^{181} \mathrm{p}$-tau, could more stably reflect pathological changes in the brain of iNPH patients [19]. Therefore, we selected CSF T-tau, ${ }^{181} p$-tau and A $\beta 1-42$ to access their predicted value of shunt responsiveness based on tap test results. Simultaneously, other influencing factors were analyzed to identify better biomarkers or combined factors for the diagnosis and prognosis assessment of iNPH patients.

The results showed that the levels of CSF biomarkers were related to tap test responsiveness. The levels of tau and its phosphorylated form in CSF of iNPH patients were decreased, especially in the tap test (+) group, although the levels of A $31-42$ did not change significantly. The reduction of CSF proteins in iNPH patients may result from the facilitated metabolic product drainage [20]. In addition, the ratio of p/T-tau in the tap test (+) group was higher, suggesting that the magnitude of $p$-tau reduction is significantly lower than T-tau, resulting in a relatively higher ratio of $p / T$-tau. Moreover, the levels of $p$-tau, the ratio of $p$ tau/AB1-42 and the responsiveness of the tap test were related to the number of combined symptoms in iNPH patients. The number of combined symptoms were also affected by the prevalence of hypertension, initial pressure and pressure difference of CSF. Alternatively, in addition to combined symptoms, a correlation was also found between the end pressure or pressure difference of CSF and tap test responsiveness, which may suggest better brain tissue compliance in patients with tap test positivity. Collectively, there is a "concentric"-like relationship between tap test responsiveness with the ratio of $p / T$ tau, pressure difference of CSF and numbers of combined symptoms, while the latter two factors were affected by hypertension, initial or end pressure of CSF.

To clarify the contribution of these related factors to tap test responsiveness, further binary logistic regression analyses were conducted. The results showed that $P$ (tap test responsiveness) $=1 / 1+e^{\wedge}$ $(-5.505+55.314$ * ratio of $\mathrm{p} / \mathrm{T}$-tau -1.586 * numbers of combined symptoms). The inferential combined indicators were calculated as $-5.505+0.553 *$ percentage of $p / T$-tau $-1.586 *$ numbers of combined symptoms. Further ROC curve results showed the combined indicators gave the highest AUC of 0.90 , and the cut-off value was -0.77 , which had a sensitivity and specificity of $94.12 \%$ and $72.73 \%$, respectively. All of the results revealed the correlation between CSF biomarkers, clinical symptoms and tap test responsiveness, which lays a research foundation for exploring the correlation as an evaluation index for 
clinical symptoms and shunt response. We further calculated the cut-off value of combined indicators with the highest sensitivity and specificity using numbers of combined symptoms and $\mathrm{p} / \mathrm{T}$-tau in the judgment of tap test responsiveness, which is beneficial for the feasibility of clinical application.

This study only shows the predictive value of the CSF biomarkers of the tap test in patients with iNPH, and its mechanism remains to be further studied. Of course, gender bias of subjects exists in this study, meaning that a larger sample is still needed. There is also a potential drawback in the setup of this study, which is the ambiguous concentration gradient of biomarkers in the $2 \times 15 \mathrm{~mL}$ CSF draw. The first milliliters of a CSF draw from iNPH patients would be considered and analyzed in future verification studies, in which the difference in levels is perhaps more pronounced, resulting in an improved diagnostic performance $[15,21]$.

\section{Conclusions}

The analysis of CSF biomarkers from iNPH patients showed a decrease of tau and its phosphorylated form, especially in the tap test (+) group, although the ratio of $p / T$-tau was relatively higher. Alternatively, the responsiveness of the tap test was related to the number of combined symptoms, end pressure and pressure difference. Further analysis showed that combined indicators $\left(-5.505+0.553^{*}\right.$ percentage of $\mathrm{p} / \mathrm{T}$ tau -1.586 * numbers of combined symptoms) gave the highest area under the ROC curve of 0.90 . The cut-off value (-0.77) was determined by maximizing Youden's Index (0.67), of which the sensitivity and specificity were $94.12 \%$ and $72.73 \%$, respectively.

\section{Abbreviations}

CSF: Cerebrospinal fluid; iNPH: Idiopathic normal pressure hydrocephalus; AD: Alzheimer's disease; MRI: Magnetic resonance imaging; ELISA: Enzyme-linked immunosorbent assay; QC: Quality control; MMSE: Mini-mental state examination

\section{Declarations}

\section{Acknowledgments}

This ELISA test was supported and sponsored by Dr. Sen Liu and his research team at Beijing Pason Pharmaceuticals Inc., including the experiment methods, diagnostic reagents and the technical support. We would like to express our sincere gratitude to Dr. Sen Liu and his team.

\section{Authors' Contributions}

Conceptualization, Rongrong Hua and Yan Xing; methodology, Yanfeng Li; software, Rongrong Hua; validation, Chunyan Liu, and Xing Liu; formal analysis, Jinwu Zhu; investigation, Jie Zhang, Lidong Wang, Zhe Shi, Jian Li, Shuangyan Kong, Chenhui Yang, Nan Liu, Lijuan Liu, Jie Sun, Qiong Yang, and Yubing Wu; resources, Ying Zhou; data curation, Rongrong Hua; writing-original draft preparation, Rongrong 
Hua; writing-review and editing, Yan Xing; supervision, Yanfeng Li; project administration, Rongrong Hua. All authors have read and agreed to the published version of the manuscript.

\section{Funding}

This work was supported by Beijing Natural Science Foundation (7202237), Hydrocephalus Laboratory Funding of Aviation General Hospital and Doctoral Research Fund (BS2019-003).

\section{Availability of data and materials}

The datasets supporting the conclusions of this article are included within the article and additional files.

\section{Ethics approval and consent to participate}

The study protocol was approved by the Ethics Committee of Aviation General Hospital. All methods were performed in accordance with the relevant guidelines and regulations. All subjects gave written informed consent for the use of clinical data for research purposes. Consent to publish has been obtained from the participant to report individual patient data.

\section{Consent for publication}

Consent to publish has been obtained from the participants.

\section{Competing Interest}

The authors declare no conflict of interest.

\section{References}

1. Liew BS, Takagi K, Kato Y, Duvuru S, Thanapal S, Mangaleswaran B. Current Updates on Idiopathic Normal Pressure Hydrocephalus. Asian journal of neurosurgery. 2019;14(3):648-56.

2. Williams MA, Malm J. Diagnosis and Treatment of Idiopathic Normal Pressure Hydrocephalus. Continuum. 2016;22(2 Dementia):579-99.

3. Graff-Radford NR, Jones DT. Normal Pressure Hydrocephalus. Continuum. 2019;25(1):165-86.

4. Halperin JJ, Kurlan R, Schwalb JM, Cusimano MD, Gronseth G, Gloss D. Practice guideline: Idiopathic normal pressure hydrocephalus: Response to shunting and predictors of response: Report of the Guideline Development, Dissemination, and Implementation Subcommittee of the American Academy of Neurology. Neurology. 2015;85(23):2063-71.

5. Toma AK, Papadopoulos MC, Stapleton S, Kitchen ND, Watkins LD. Systematic review of the outcome of shunt surgery in idiopathic normal-pressure hydrocephalus. Acta Neurochir (Wien). 2013;155(10):1977-80.

6. Abu Hamdeh S, Virhammar J, Sehlin D, Alafuzoff I, Cesarini KG, Marklund N. Brain tissue A $\beta 42$ levels are linked to shunt response in idiopathic normal pressure hydrocephalus. Journal of Neurosurgery. 
2018:1-9.

7. Jingami N, Asada-Utsugi M, Uemura K, Noto R, Takahashi M, Ozaki A, et al. Idiopathic normal pressure hydrocephalus has a different cerebrospinal fluid biomarker profile from Alzheimer's disease. Journal of Alzheimer's disease : JAD. 2015;45(1):109-15.

8. Relkin N, Marmarou A, Klinge P, Bergsneider M, Black PM. Diagnosing idiopathic normal-pressure hydrocephalus. Neurosurgery. 2005;57(3 Suppl):S4-16; discussion ii-v.

9. Mori E, Ishikawa M, Kato T, Kazui H, Miyake H, Miyajima M, et al. Guidelines for management of idiopathic normal pressure hydrocephalus: second edition. Neurologia medico-chirurgica. 2012;52(11):775-809.

10. Dubois B, Feldman HH, Jacova C, Hampel H, Molinuevo JL, Blennow K, et al. Advancing research diagnostic criteria for Alzheimer's disease: the IWG-2 criteria. The Lancet Neurology. 2014;13(6):61429.

11. Olsson B, Lautner R, Andreasson U, Ohrfelt A, Portelius E, Bjerke M, et al. CSF and blood biomarkers for the diagnosis of Alzheimer's disease: a systematic review and meta-analysis. The Lancet Neurology. 2016;15(7):673-84.

12. Riemenschneider M, Wagenpfeil S, Vanderstichele H, Otto M, Wiltfang J, Kretzschmar H, et al. Phospho-tau/total tau ratio in cerebrospinal fluid discriminates Creutzfeldt-Jakob disease from other dementias. Molecular psychiatry. 2003;8(3):343-7.

13. Ritchie C, Smailagic N, Ladds EC, Noel囚torr AH, Ukoumunne O, Martin S. CSF tau and the CSF tau/ABeta ratio for the diagnosis of Alzheimer's disease dementia and other dementias in people with mild cognitive impairment (MCl). Cochrane Database of Systematic Reviews. 2013(10).

14. Jeppsson A, Zetterberg H, Blennow K, Wikkelso C. Idiopathic normal-pressure hydrocephalus: pathophysiology and diagnosis by CSF biomarkers. Neurology. 2013;80(15):1385-92.

15. Graff-Radford NR. Alzheimer CSF biomarkers may be misleading in normal-pressure hydrocephalus. Neurology. 2014;83(17):1573-5.

16. Jingami N, Uemura K, Noto R, Asada M, Kinoshita A. Idiopathic Normal Pressure Hydrocephalus has a Different Cerebrospinal Fluid Biomarker Profile from Alzheimer's Disease. 2015.

17. Chen Z, Liu C, Zhang J, Relkin N, Xing Y, Li Y. Cerebrospinal fluid Abeta42, t-tau, and p-tau levels in the differential diagnosis of idiopathic normal-pressure hydrocephalus: a systematic review and metaanalysis. Fluids and barriers of the CNS. 2017;14(1):13.

18. Craven CL, Baudracco I, Zetterberg H, Lunn MPT, Chapman MD, Lakdawala N, et al. The predictive value of T-tau and AB1-42 levels in idiopathic normal pressure hydrocephalus. Acta Neurochirurgica. 2017.

19. Herukka SK, Rummukainen J, Ihalainen J, von Und Zu Fraunberg M, Koivisto AM, Nerg O, et al. Amyloid-beta and Tau Dynamics in Human Brain Interstitial Fluid in Patients with Suspected Normal Pressure Hydrocephalus. Journal of Alzheimer's disease : JAD. 2015;46(1):261-9.

20. Xie L, Kang H, Xu Q, Chen MJ, Liao Y, Thiyagarajan M, et al. Sleep drives metabolite clearance from the adult brain. Science. 2013;342(6156):373-7. 
21. Jingami N, Uemura K, Asada-Utsugi M, Kuzuya A, Yamada S, Ishikawa M, et al. Two-Point Dynamic Observation of Alzheimer's Disease Cerebrospinal Fluid Biomarkers in Idiopathic Normal Pressure Hydrocephalus. Journal of Alzheimer's disease : JAD. 2019;72(1):271-7.

Figures

\section{Identified $\mathrm{n}=42$ samples}

\section{3 sample invalid}

\section{Analysis $\mathrm{n}=39$ samples}

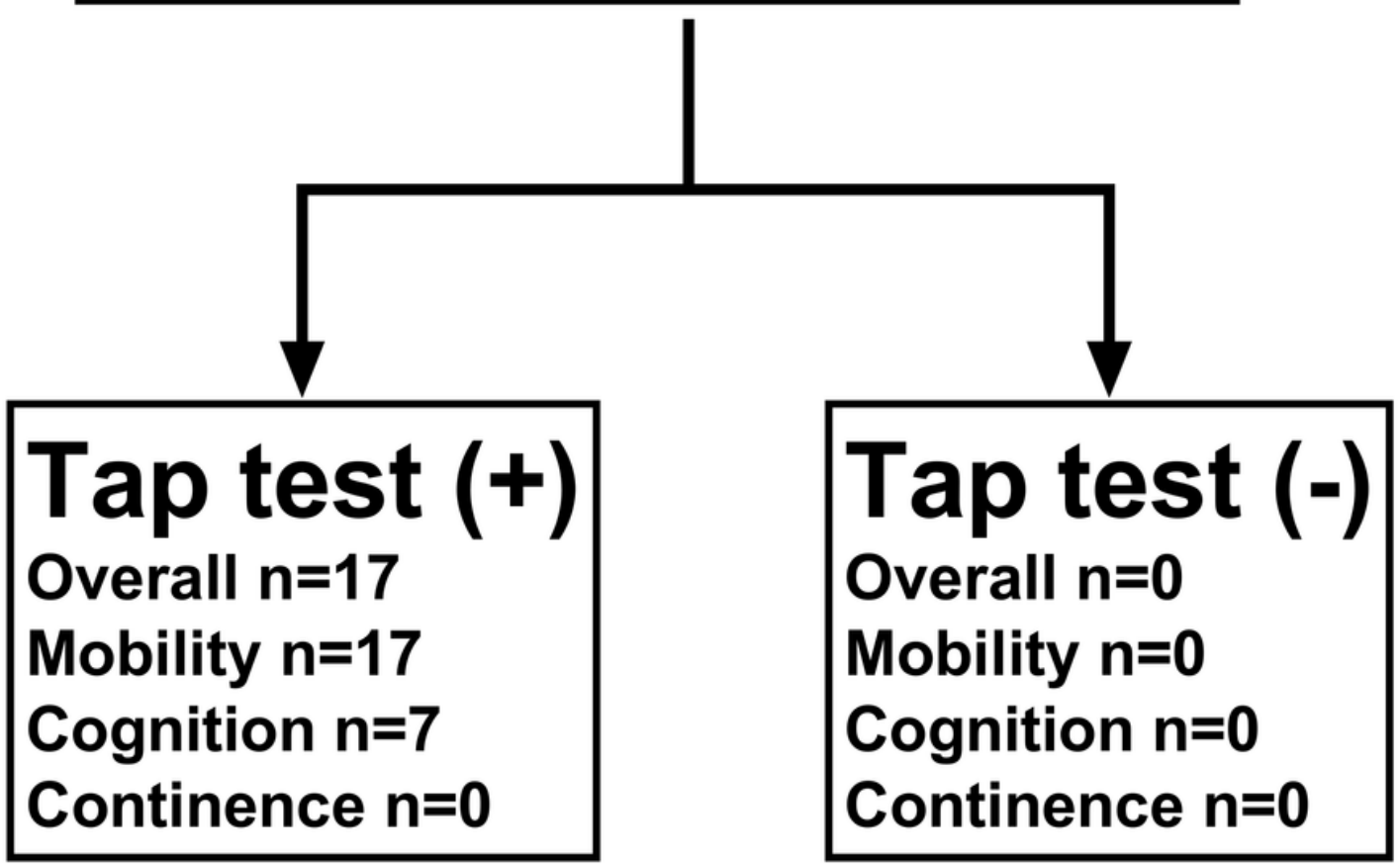

Figure 1

Flow chart of patient participants. A total of 39 suspected iNPH were included in the final predictive value analysis. Secondary samples with tap test $(+)(n=17)$ or $(-)(n=22)$ were included in the longitudinal analysis of biomarkers in lumbar CSF. 

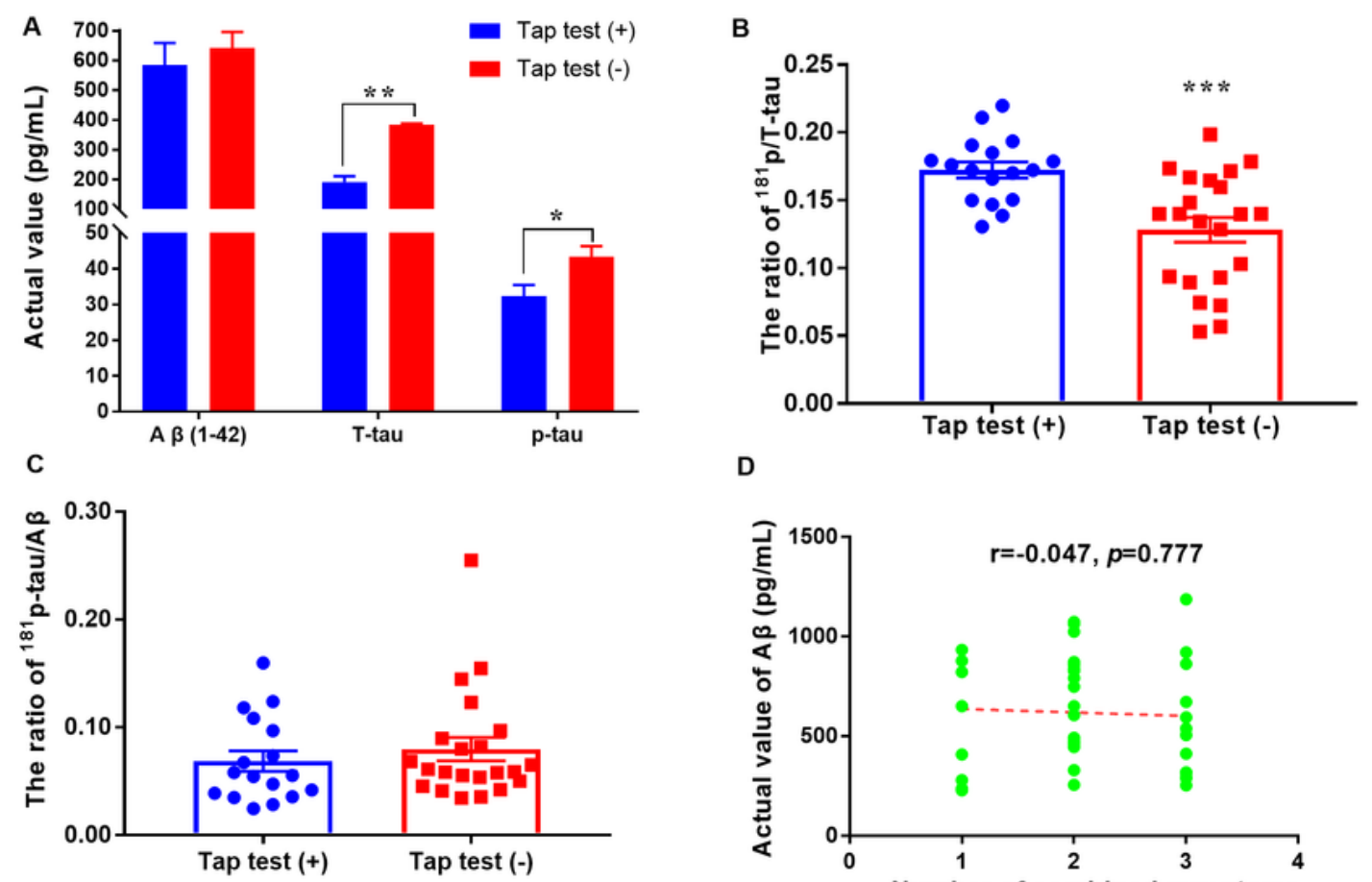

D
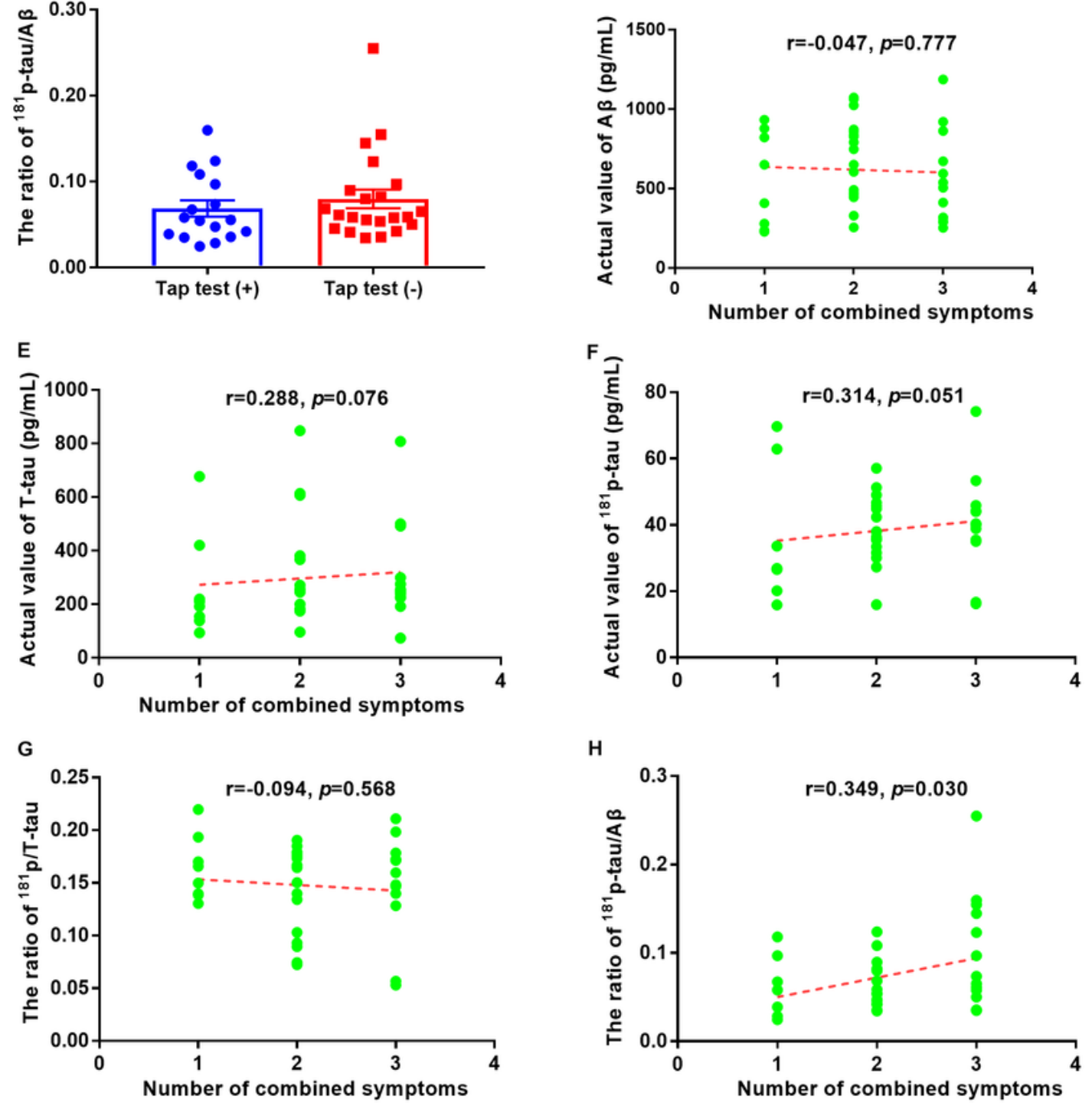

\section{Figure 2}

$A \beta 1-42$, tau levels and relationship with tap test response or combined symptoms in iNPH patients. A. The levels of CSF A 1-42, T-tau, and p-tau between tap test (+) and (-) group. ( ${ }^{*} p<0.05$ and $* * ~ p<0.01$ versus corresponding group) B. The ratio of $181 \mathrm{p} / \mathrm{T}$-tau in the tap test $(+)$ and $(-)$ group. $\left({ }^{* \star *} \mathrm{p}<0.001\right.$ versus corresponding group) $\mathrm{C}$. The ratio of $181 \mathrm{p}$-tau/Aß1-42 in the tap test (+) and (-) group. D-H. The 
correlation analysis between the actual values of $A \beta 1-42$, T-tau, $p$-tau, $p / T$-tau and $p$-tau/A 1 1-42 with the numbers of combined symptoms.
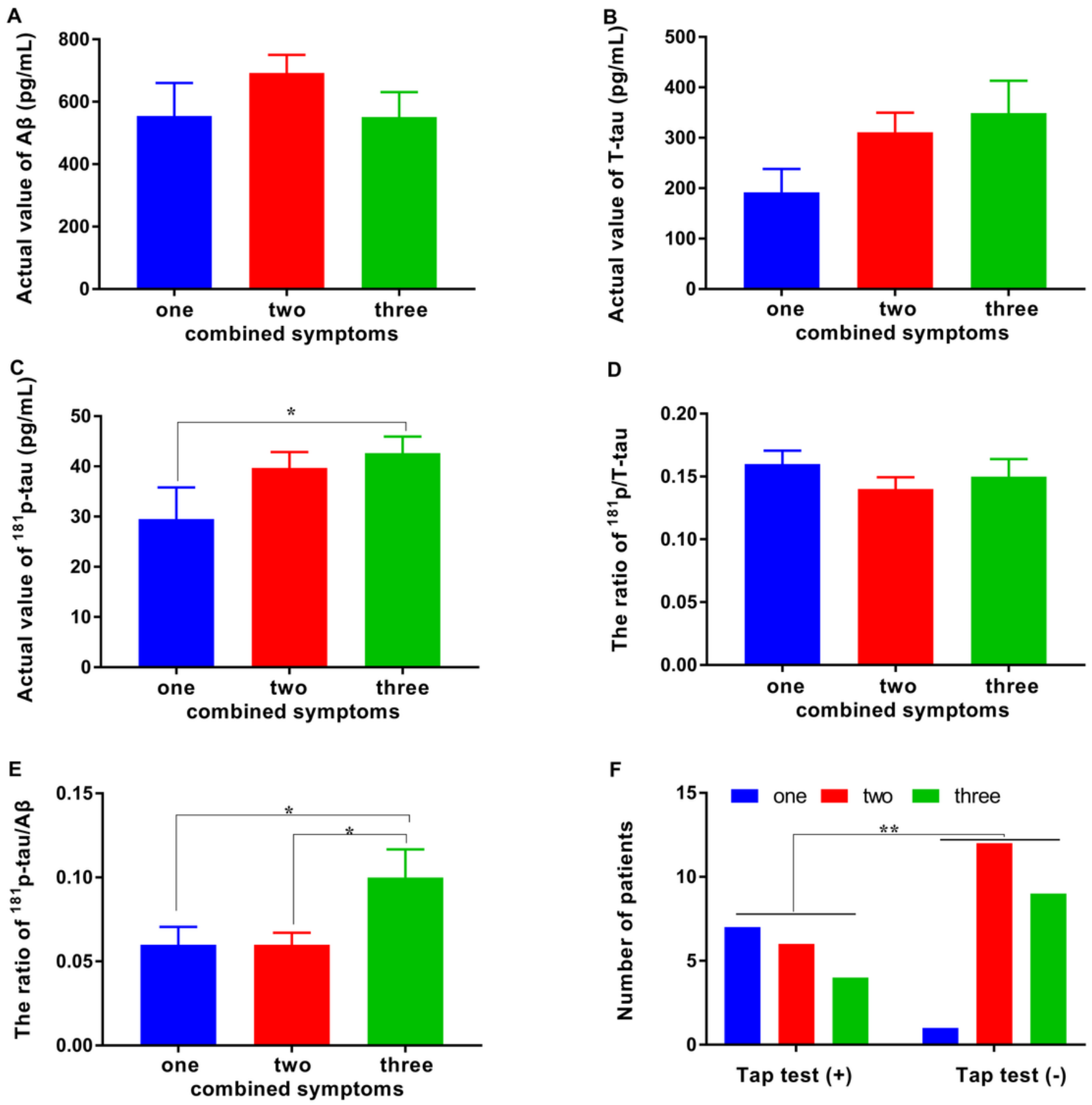

Figure 3

Differences of CSF biomarker and tap test responsiveness between subgroups with different combined symptoms. A-E. Differences of AB1-42, T-tau, 181p/T-tau, 181p-tau/AB1-42 between the subgroups with different numbers of combined symptoms. $F$. The relationship of the tap test responsiveness with number of combined symptoms. ( ${ }^{*}<0.05$ and ${ }^{*} p<0.01$ versus corresponding group) 

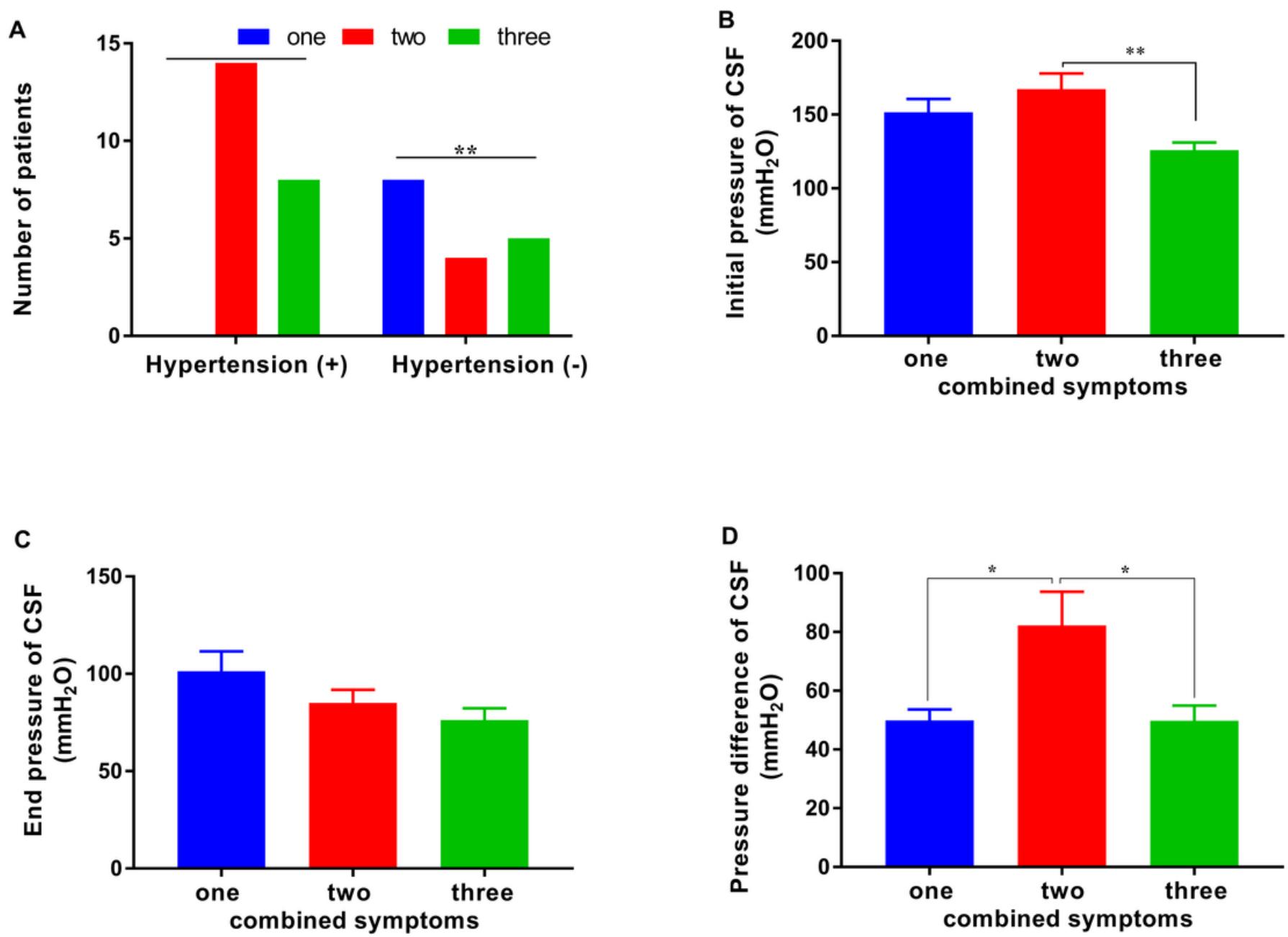

Figure 4

The distribution of combined symptoms and the correlated factors. A. The distribution of hypertension in patients with one/two/three combined symptoms. B-D. The initial pressure, end pressure and pressure difference of CSF in patients with one/two/three combined symptoms. (* $p<0.05$ and ${ }^{* \star} p<0.01$ versus corresponding group) 

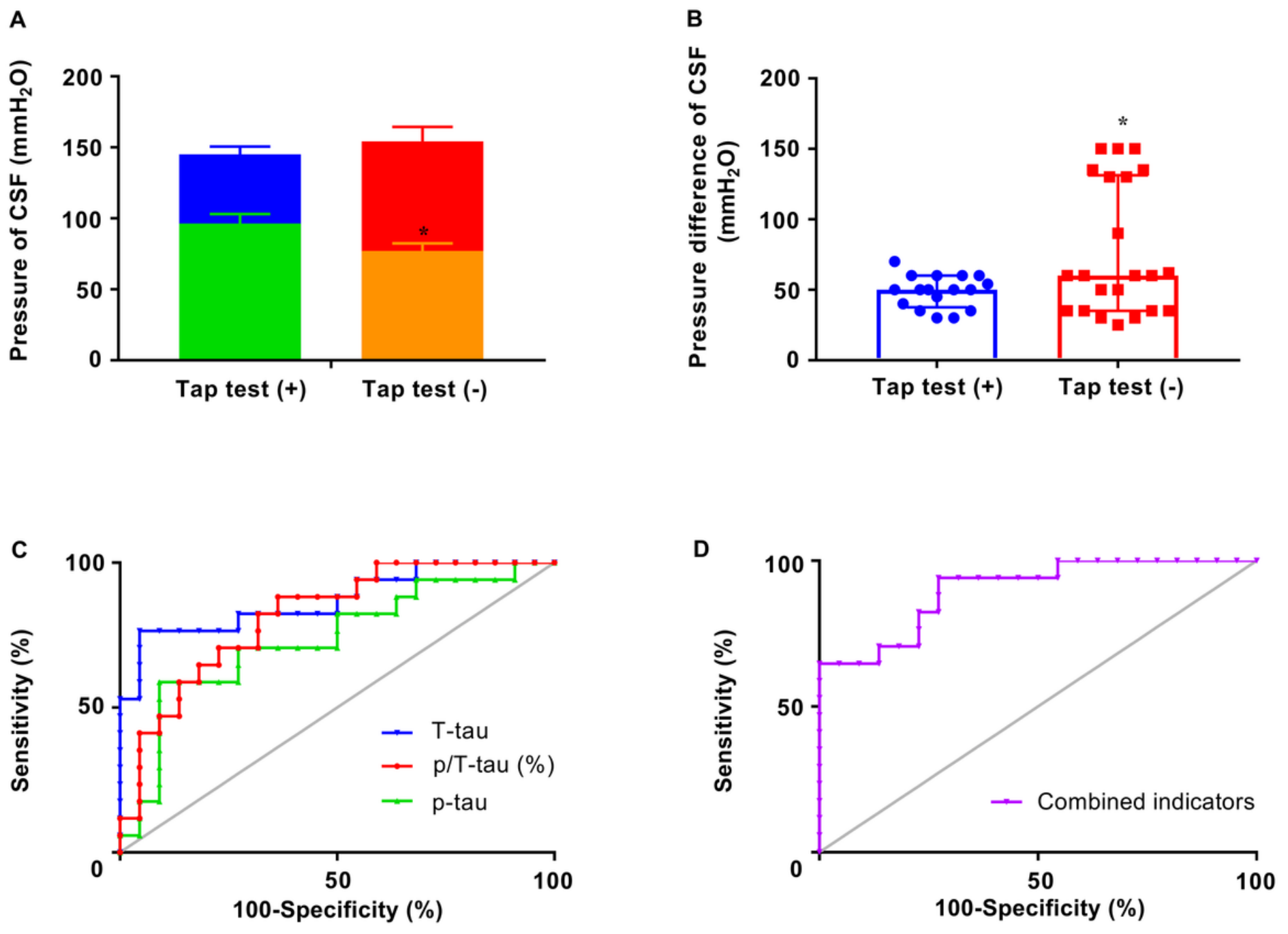

Figure 5

The responsiveness of the tap test and its correlated factors. A. The difference of initial and end pressure of CSF in patients with different tap test responsiveness. B. The pressure difference of CSF in patients with different tap test responsiveness. C. ROC curves of T-tau, $181 \mathrm{p}$-tau, and $181 \mathrm{p} / \mathrm{T}$-tau in accessing tap test responsiveness. D. ROC curves of combined indicators $(-5.505+0.553 *$ percentage of $p / T$-tau -1.586 * numbers of combined symptoms) in accessing tap test responsiveness. ( ${ }^{*} p<0.05$ versus corresponding group) 